veränderlicher Sterne sich von vorausgefassten Meinungen über den Gang des Lichtwechsels unabhärigig zu machen, und bei den Algolsternen wird diese Schwierigkeit noch viel grösser, da es unmöglich ist, die an demselben Abend schon angestellten Beobachtungen zu vergessen. Wenn ausserdem, wie bei Y Cygni, die ganze Amplitude klein ist, und die zu Gebote stehenden Vergleichsterne nicht so passend sind, wie wünschenswerth, muss die Unsicherheit noch grösser werden. Ich sehe keinen Weg sich davon unabhängig zu machen, als wenn man entweder die Minima durch Photo- graphie bestimmen könnte oder, was vielleicht noch besser wäre, wenn sowohl in Europa, wie in Amerika möglichst viele Beobachter sich mit diesem Stern beschäftigen wollten, was, da der Stern nunmehr besonders interessant geworden ist, gehofft werden könnte. Jedenfalls wird die Ursache, welche wohl bisher in erster Linie die Beobachtungen beeinträchtigt hat, nämlich die Unmöglichkeit, mit genügender Sicherheit die Minima im voraus berechnen $z u$ können, nunmehr nicht vorhanden sein."

L.

\title{
Ueber die Karlsruher Beobachtungen von Mösting A.
}

Auf die Aufforderung in Nr. 2917 der Astr. Nachr. hat Herr Dr. Boy Matthiessen in Karlsruhe in verdienstvoller Weise Culminationen der Kraters und des Mondrandes beobachtet und 5 Culminationen in $\mathrm{Nr} .2966,16$ in Nr. 3078 der Astr. Nachr, veröffentlicht. Diese erste Reihe von Meridianbeobachtungen des Kraters ist insofern von besonderem Interesse, als sie Material liefert zur Beurtheilung der Frage, wie die Ephemeriden den Ort des Kraters darstellen. Auch hat Herr Dr. Matthiessen die Beobachtungen bereits mit der Mondephemeride des Nautical Almanac verglichen, doch sind noch zwei Punkte bei der Reduction zu berücksichtigen. Zunächst sind in der Zeit 1890 Febr. 27 bis Mai 5 die Differenzen Mondmittelpunkt-Krater, wie sie für Greenwich gelten, benutzt, während sie für den Meridian von Karlsruhe zu interpoliren sind. Zweitens giebt die Ephemeride des Kraters, wie aus den Gleichungen hervorgeht, den Unterschied der Rectascension und Declination des Mondes und Kraters zur Zeit der Culmination des Mondes an. Beobachtet wird dagegen die Rectascension zur Zeit der Culmination des Kraters. Man hat also die Eigenbewegung in der $Z$ wischenzeit hinzuzufügen, ebenso wie man den Radius und die Durchgangszeit desselben unterscheidet. Hiermit erhält man folgende Unterschiede im Sinne Beobachtung - Nautical Almanac:

$\begin{array}{rcccc}1890 & \text { Wahre Zeit } & \text { Rand } & \text { Krater } & \text { Rand-Krater } \\ \text { Febr. } 2 & 10^{\mathrm{h}} .4 & \text { I }+0.04 & +0.05 & -0.01 \\ 4 & 12.0 & \text { I }-0.26 & -0.18 & -0.08 \\ 5 & 12.8 & \text { II }-0.09 & -0.16 & +0.07 \\ 9 & 15.7 & \text { II }+0.05 & +0.03 & +0.02 \\ 10 & 16.5 & \text { II }-0.09 & -0.01 & -0.08\end{array}$

\begin{tabular}{|c|c|c|c|c|c|c|}
\hline 189 & & Wahre Zeit & & Rand & Krater & Kand-Krater \\
\hline Febr. & & $6^{\mathrm{h}} \cdot 7$ & I & $+0^{5} .49$ & -0.6 I & $+15^{5} 10$ \\
\hline & 28 & $7 \cdot 5$ & I & -0.30 & -0.30 & 0.00 \\
\hline März & $\mathbf{I}$ & 8.4 & I & 十0.1 I & +0.15 & -0.04 \\
\hline & 4 & 10.8 & I & +0.12 & +0.21 & -0.09 \\
\hline & 29 & 7.2 & I & +0.04 & +0.16 & -0.12 \\
\hline & 31 & 8.8 & 1 & +0.01 & +0.04 & -0.03 \\
\hline April & 2 & 10.3 & I & -0.04 & +0.01 & -0.05 \\
\hline & 4 & I 1.8 & I & -0.05 & +0.08 & -0.13 \\
\hline Mai & 3 & 11.8 & I & -0.09 & -0.31 & +0.22 \\
\hline & 4 & I 2.1 & I & +0.02 & +0.13 & $\cdots-0.1 \mathrm{I}$ \\
\hline 189 & $1^{5}$ & 13.1 & II & 0.00 & -0.06 & +0.06 \\
\hline Febr, I & I 7 & $7 \cdot 4$ & I & -0.66 & -0.73 & +0.07 \\
\hline & 19 & 9.1 & I & -0.23 & -0.18 & -0.05 \\
\hline & 20 & 9.9 & I & -0.15 & 0.00 & -0.15 \\
\hline & $2 I$ & 10.7 & I & -0.09 & -0.11 & +0.02 \\
\hline & 28 & 15.7 & II & +0.21 & +0.08 & +0.13 \\
\hline
\end{tabular}

Die Abweichungen vom Nautical Almanac haben beim Rand und beim Krater meist dasselbe Vorzeichen und die Differenz Rand-Krater zeigt, dass die Ephemeride den Ort des Kraters wenigstens innerhalb der Grenzen der Beobachtungsfehler darstellt. Unter der Annahme, dass 1890 Febr. 27 bei der Abzählung oder Reduction ein Versehen von $\mathrm{r}^{\mathrm{s}}$ vorliegt, wird das Mittel der Differenzen

Rand-Krater $=-0.012 \pm 0.021$ mittl. Fehl.

Eine graphische Darstellung dieser Differenzen als Function der wahren Zeit oder der Phase lässt keinen systematischen Gang erkennen.

\section{Franz.}

\section{Die Anzahl der Sterne auf einigen photographischen Aufnahmen.}

Von der Umgebung von $a$ Cygni besitze ich drei mit dem 5 zölligen Kranz-Aplanat gemachte Aufnahmen:

$$
\begin{aligned}
& \text { B } 302 ; 1891 \text { Sept. } 7 \text {, von } 1 \text { ho Belichtung }
\end{aligned}
$$

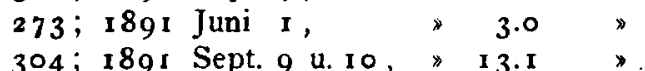

Es bot sich mir daher die Gelegenheit, Zählungen der Sterne vorzunehmen, um Material über den Sternzuwachs in dieser Gegend bei längerer Belichtung zu erhalten.

Um die Sterne auf einem Theil dieser Platten zu zählen - alle zu zählen wäre etwas zeitraubend - wurde ein Netz unter die Platten gelegt, so dass jede Stelle des Gitters genau an die correspondirende Stelle auf den drei Platten zu liegen kam, und also ein Quadrat des Gitters auf jeder Platte die nämliche Stelle des Himmels umfasste. Die Seitenlänge eines Quadrats betrug $0.462 \mathrm{~cm}$, was bei dem benutzten Objectiv einem Bogen von $0^{\circ} 20^{\circ}$ entspricht.

Es wurden nun auf den drei Platten die Sterne in einer Reihe solcher Quadrate gezählt, und zwar wurden zehn Quadrate in einer mittelreichen-Gegend, zehn in einer sternarmen Gegend und zehn in einer sternreichen Gegend gewählt. In der folgenden Tabelle sind die Resultate dieser 
Zählung zusammengestellt, und es stehen immer die Zahlen der Sterne im selben Quadrat der drei Platten neben einander. Ich gebe die Einzelzahlen, weil ihr Verlauf trotz grosser Abweichungen doch interessante Analogien enthält:

\begin{tabular}{|c|c|c|c|}
\hline $\begin{array}{c}\text { Nr. der } \\
\text { Quadrate }\end{array}$ & $I^{h}$ & $3^{h}$ & $I 3^{h}$ \\
\hline I & 35 & 151 & 219 \\
\hline 2 & 30 & 120 & 205 \\
\hline 3 & 47 & 92 & 198 \\
\hline 4 & 45 & 121 & 220 \\
\hline 5 & 41 & 109 & 176 \\
\hline 6 & 48 & 88 & 255 \\
\hline 7 & 20 & 113 & 210 \\
\hline 8 & 48 & 170 & 222 \\
\hline $\begin{array}{r}9 \\
10 \\
\end{array}$ & $\begin{array}{l}56 \\
38 \\
\end{array}$ & $\begin{array}{r}122 \\
82 \\
\end{array}$ & $\begin{array}{r}243 \\
170 \\
\end{array}$ \\
\hline II & 27 & 67 & 123 \\
\hline 12 & 34 & 66 & 145 \\
\hline 13 & 31 & 70 & 130 \\
\hline 14 & 15 & 40 & 142 \\
\hline I 5 & 32 & 62 & 96 \\
\hline 16 & 19 & 24 & 59 \\
\hline 17 & 18 & $4^{8}$ & 88 \\
\hline 18 & 19 & 37 & 96 \\
\hline 19 & 22 & 50 & 88 \\
\hline 20 & 13 & 48 & III \\
\hline $\begin{array}{l}21 \\
22\end{array}$ & $\begin{array}{r}118 \\
87\end{array}$ & $\begin{array}{l}185 \\
182\end{array}$ & $\begin{array}{l}303 \\
282\end{array}$ \\
\hline 23 & $9 I$ & 182 & 392 \\
\hline 24 & I 3 I & 165 & 288 \\
\hline 25 & 105 & 175 & 305 \\
\hline 26 & 76 & I 19 & 210 \\
\hline 27 & 46 & 98 & 230 \\
\hline 28 & 86 & 172 & 321 \\
\hline 29 & 77 & 136 & 233 \\
\hline 30 & 102 & 149 & 240 \\
\hline me $3^{\circ}$ & 1557 & 3243 & 5900 \\
\hline
\end{tabular}

Es beträgt also die durchschnittliche Dichtigkeit der Sterne auf dem Quadrat von $20^{\circ}$ Seite auf den drei Platten: und auf dem Quadratgrad:

$$
52,108, \quad 197
$$

$$
467,973, \quad \text { r } 770 \text {. }
$$

Die Platten haben eine belichtete Oberfläche von fast genau 1000 Quadraten. Es sind daher schätzungsweise :

$$
\begin{aligned}
& \text { auf der I-stiundigen Platte } 52000 \text { Sterne }
\end{aligned}
$$

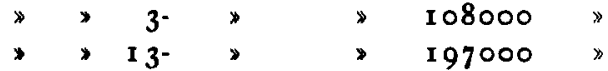

Die Anzahl der Sterne auf der Durchmusterungskarte ist in selben Raum etwa 3500 .

Würden bei den länger exponirten Platten die helleren Sterne nicht mehr und mehr schwächere überdecken, so wäre die Anzahl auf der Platte noch bedeutend grösser. Da aber hierfür jede Regel fehlt, so müssen wir uns mit den obigen Zahlen begnügen.

Aus den Schätzungen nach der Sichtbarkeit der photographirten Sterne in seinem $17 \frac{1}{4}$ Zöller glaubt Rev. Espin annehmen zu können, dass etwa noch Sterne bis zur 15. Grösse der Argelander'schen Scala auf der Reproduction der 13-stündigen Platte sichtbar seien. Dann müssten auf der Platte selbst sicher noch Sterne unter der 15. Grösse der Argelander'schen Scala vorhanden sein, denn durch den Druck geht mindestens eine Grössenclasse verloren.

Aehnliche Resultate erhielt ich von drei Platten der Plejadengegend, die $1^{\mathrm{h}}, 2^{\mathrm{h}}$ und $6^{\mathrm{h}}$ exponirt sind. In 20 Quadraten wurden hier die Sternzahlen gefunden:

$$
\text { 294, 582, 833, }
$$

während die Reproduction der Herren Henry auf demselben Raum etwa 900 Sterne zeigt.

Auf der I-stündigen Platte sind noch die Sterne I 1 .5 vorhanden, z. B. die Sterne (82), (314), (325). Auf der 2-stündigen Platte sind noch die von Charlier als 13.5 geschätzten Sterne gut sichtbar, wie z. B. die Sterne (35 I), (343), (489), (424). Auf der 6-stündigen Platte endlich sind alle die von Charlier als $14^{\mathrm{m}}$ geschätzten Sterne noch vorhanden und fast alle auf der Pariser Reproduction als $5^{\mathrm{m}}$ bis $16^{\mathrm{m}}$ bezeichneten.

Heidelberg, Sternwarte, I 89 I October. Max Wolf.

\section{Ueber den Cometen des Jahres 1689.}

\section{Von Dr. F. Holetschek.}

(Auszug aus einer der kais. Akademie der Wissenschaften in Wien am ro. December r8gr vorgelegten Abhandlung.)

Dieser durch einen langen, gekrümmten Schweif ausgezeichnete Comet wurde im December 1689 am Morgenhimmel gesehen, als er durch das Sternbild des Wolfes in südlicher Richtung gegen $\alpha$ Centauri ging. Die neue Untersuchung seiner Bahn ist hauptsächlich dadurch veranlasst worden, dass die Bemerkung der Beobachter in Malaka, die vom Cometen durchlaufene Strecke sei vom 14. bis zum 15. December am grössten, nämlich ein wenig mehr als $3^{\circ}$ gewesen und von da an mit jedem Tag kleiner geworden, durch keine der bisher berechneten Bahnen dar. gestellt wird. Die Bahn von Pingré entfernt sich von dieser
Bemerkung noch am wenigsten, indem sie dem Cometen eine fast gleichförmige Bewegung und zwar für jeden Tag nahe $2^{\circ}$ gibt; die anderen Bahnen, nämlich die von Peirce (A. N. Bd. 20 p. 396), Vogel (Monthly Notices Vol. 12 p. 206 und A. N. Bd. 34 p. $3^{87}$ ) und die Supposition der Bahn eines zur Gruppe I 843 I, 1880 1, I882 II gehörenden Cometen, liefern alle eine zunehmende Geschwindigkeit.

In der Abhandlung sind nun aus verschiedenen Combinationen der von den Beobachtern gemachten Angaben, sowie auch anderer, durch erweiterte Deutung des Textes gewonnener Rechnungsgrundlagen Parabeln abgeleitet, um 